\title{
A Chatbot for supporting users in Cultural Heritage contexts
}

\author{
Fabio Clarizia \\ DIIn \\ University of Salerno \\ Fisciano (Salerno), Italy \\ fcolarizia@unisa.it
}

\author{
Francesco Colace \\ DIIn \\ University of Salerno \\ Fisciano (Salerno), Italy \\ fcolace@unisa.it
}

\author{
Marco Lombardi \\ DIIn \\ University of Salerno \\ Fisciano (Salerno), Italy \\ malombardi@unisa.it
}

\author{
Domenico Santaniello \\ DIIn \\ University of Salerno \\ Fisciano (Salerno), Italy \\ dsantaniello@unisa.it
}

\begin{abstract}
The distance education of today is performed through new technologies, which assist whit the aim to improving the educational process. New technologies brought elearning environments more and more complete and able to supply learning experiences increasingly similar to traditional educational processes. The introduction of chatbots could represent an important aspect of the students' educational process. Chatbots are particular applications able to simulate typical human being conversations; through inference engines and modern Natural Language Processing techniques, a chatbot could be able to provide tailored educational paths. In this paper is presented a chatbot prototype in order to support students in the study of Cultural Heritage. This system takes advantage of inference techniques and approaches based on Natural Language Processing in order to provide a fluid conversation responding to the student's needs. The real experimentation was performed in the application context of the archaeological park of Paestum, where students were able to experience contextualized and personalized learning paths based on the recommendation capacity of the proposed system. In terms of student satisfaction, our proposed system shows very satisfactory results.
\end{abstract}

\section{Keywords-Chatbot, Technology-Mediated Learning,} Cultural Heritage

\section{INTRODUCTION}

The world of distance education is enriching with new tools able to improve the training process. Modern e-Learning environments do not simply aim to reproduce the typical processes of the world of traditional education but seek to enrich them thanks to the support of new technologies. In this scenario, the introduction of the chatbots could be an effective enhancement for the distance learning process [1]. Chatbots, in fact, are becoming one of the most interesting approach in many fields: their design has become increasingly sophisticated and their use adopted commerce, entertainment, public sector, social networks and education [2] [4]. In fact, the introduction of chatbots comes with four main advantages. Firstly, the implementation of chatbots saves customer service costs by replacing nearly all human assistants. Secondly, chatbots increase user satisfaction by speeding up response times and being available twenty-four hours a day. Thirdly, chatbots can be proactive: they can anticipate user questions and needs. Fourthly, chatbots are also a powerful analysis tools, since conversations between users and chatbots can be analysed to better understand customer requirements [5] [6].

In distance learning field, chatbots promise real enhancements in the learning process and a real improvement in the field of Intelligent Tutoring Systems (ITS) [7] [8] [9].
The ITS are environments that incorporate artificial intelligence and support the students' learning process. These tools, generally, are limited to specific knowledge domains. Unlike ITS, chatbots focus on conversation and are able to analyse the student's learning context and needs. On the other hand, teaching is a relational act based on communication and interaction and chatbots have significant educational potential thanks to their communicative ability through natural language. This kind of approach is effective especially in large-scale learning scenario where the problem of individual student support is important. Another impressive feature of the chatbots is in their efficiency: they can operate every time supporting students everywhere and allow teachers to avoid answering repetitive questions [10].

Despite all these advantages, the diffusion of chatbots in the education field is still low: a small number of researches have shown experiences in learning scenarios [11] [12]. A lot of them have been developed on IBM's Watson Platform [13] while other ones are created with specific tools. Chatbots in education can achieve two main aims [1]:

- $\quad$ To support students in tackling different topics and reflect amongst themselves on the basis of starting questions posed by the chatbot

- To infer information about the students' perceptions of a specific topic, interaction, situation or context.

Depending on the tasks carried out by chatbots in the education field, a taxonomy can be introduced [14]:

- Administrative and management tasks to foster personal productivity

- FAQs Management

- $\quad$ Student Mentoring

- Motivation

- $\quad$ Practice of specific skills and abilities

- $\quad$ Simulations

- Reflection and Metacognitive Strategies

- $\quad$ Student Learning Assessment

In this paper a chatbot for Cultural Heritage Learning, is introduced. The aim of this tool is supporting students in the learning of topics related to the Cultural Heritage field according an approach based on practice of specific skills and abilities, simulations and assessment [15][16][17]. In particular, the proposed approach adopts Natural Language Process methodologies and Context Aware Techniques for 
inferring services and contents suitable for the students' needs. Thanks to probabilistic approach as the Latent Dirichlet Allocation [18] the proposed prototype can infer from the conversation The chatbot works at its best in operative scenarios as museums or archaeological parks: in these contexts, the proposed chatbot, starting from cultural artefact, can furnish adaptive contents and enrich the learning experience of the students [19]. The paper has the following organization: in the next section more details about chatbots will be furnished. The third section will show the methodology and the architecture of the chatbot. The description of the experimental results and the conclusions will end the paper.

\section{Chatbots: MAIn CONCEPTS}

Chatbots are applications that can interact with people using language-based interfaces [20] [21]. Their purpose is to simulate a human conversation so that the speaker has an experience similar to a conversation via text or voice interactions with a real person [22] [23]. This definition includes all kinds of software enabling humans to have a conversation with a computer. In this way, virtual assistants as Amazon's Alexa, Google's Home or Apple's Siri can be considered chatbots. In literature, the first chatbot was Eliza: it analysed input sentences and created its response based on reassembly rules associated with a decomposition of the input [24]. Other interesting experiences related to the chatbots' development was Converse, Jabberwacky and Alice [25]. In particular, Alice adopts the AIML (Artificial Intelligence Mark-Up Language) allowing the introduction of Data Mining techniques. In the last period the introduction of more sophisticated Data Mining methodologies, based on Artificial Intelligence, of probabilistic methods and semantic tools has improved the skills of chatbots and their capacity to make decisions.

Generally, a chatbot has these main components:

- A Conversational Artificial Intelligence Module: this module is the Natural Language Processing engine that allows the building of the conversation. The first chatbots focused on the interpretation and recognition of patterns and rules. The modern chatbots adopt techniques based on Artificial Intelligence or deep learning approach for generating suitable responses.

- User Experience Module: this module allows a natural and coherent conversation among users and chatbot

- User Interface: this module defines the interface that user adopts for seeing or hearing the conversation with the chatbot

- Conversational design: this module allows the addition of the human logic to the artificial interaction human-machine.

The huge increase of chatbots in the last years defines three main dimensions to classify them. The first one can be defined as the "building approaches" that distinguishes between retrieval-based models and generative models. The chatbots based on retrieval-based models pick the appropriate response from a repository of predefined responses. Usually they adopt rule-based approach. Generative models generate responses out of the input with the help of machine learning techniques. This let the chatbot feel more human and capable of longer dialogs [26]. The second dimension is related to the input mode of chatbot. Users can interact with the chatbot in different ways depending on the communication interface. Three main approaches can be considered:

- Chatterboxes: users can interact with the chatbot through text or voice inputs and outputs.

- Embodied conversational agents: the interface is represented by an avatar which interacts with the user. This kind of approach involves audio, text, multimedia.

- Physical: in this case user interact with a chatbot that is embedded in a robot

The third dimension addresses the inclusion of contextual information such as linguistic and physical context in order to select the right responses.

The proposed Chatbot adopts a generative contextual model and adopts as the input mode text and voice. In the next section, more information about the chatbot architecture will be furnished.

\section{ARCHITECTURE AND APPROACH}

Before introducing the Architecture of the chatbot an example of its operation workflow. Imagine a student, which have to deepen the Greek culture in southern Italy and visit the Archaeological Park of Paestum. According to the context, the chatbot begins to provide generic content on the presence of the Greeks in southern Italy. Arriving near the temples it will begin to provide more details on the cults of the gods in Paestum. Depending on the student's requests, the chatbot will provide new contents. If for example the student will show interest in the cult of Athena, the chatbot will invite him to go to the museum in the park to view the weapons present at the time in Paestum. If the student was interested in the cult of the dead, he would be invited to see the famous Tomb of the Diver. Obviously, at regular intervals the chatbot, with some tests, will verify the student's learning. Furthermore, the chatbot is able to retrieve information [27], thanks to REST services [28], from environments such as Europeana [29] (https://pro.europeana.eu/) or DatabencArt (https://www.databencart.it/) that contain information relating to the world of cultural heritage.

The architecture of the proposed chatbot is based on some main modules, as shown in figure 1 . The storytelling module is closely related to the bot ability to guide the user through the whole learning experience, making the way of proceeding while leaving the user free to express himself and immerse himself in the personalized story of a place [30].

Each user acts differently from the others, becoming part of a creative process and creating a unique and unrepeatable visit. In planning an itinerary and in designing the narration, therefore, not all progress must be defined but a plurality of scenarios must be prepared that the user can explore freely up to crucial moments, common to all, or almost all, of the scenarios. The Context-Aware Manager deals with representing all the possible contexts of use through the Context Dimension Tree and performing contextualized queries. 


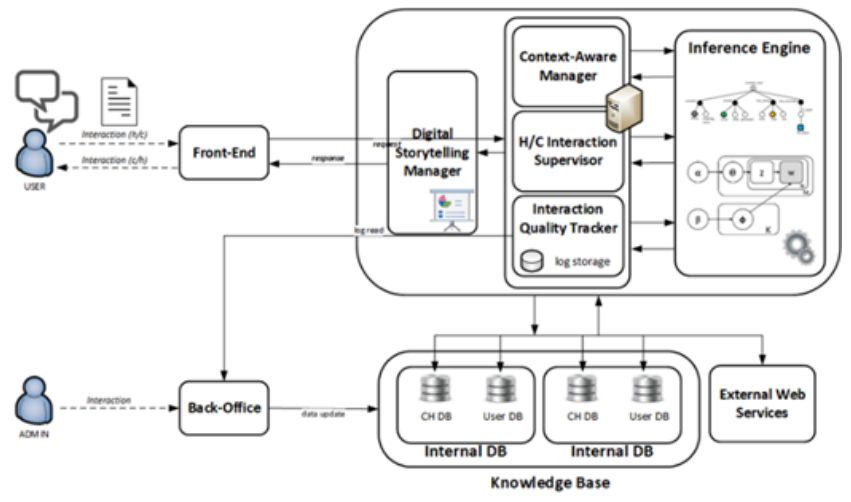

Fig. 1. The Proposed Architecture

In this way, it is possible to extract and provide personalized information by aggregating and custom-tailoring data and services extracted from different sources. Some resources are private (internal resources) or managed directly by the chatbot provider. These resources can be used, for example, to maintain the profiles of registered users, or the data of the museums reviewed. The chatbot can also interface with external services (external resources) [31].

Other modules, such as the Human/Computer Interaction Supervisor and the Interaction Quality Tracker, have the following objectives:

- $\quad$ supervise the dialogue, keeping track of timing of interaction, identifying ambiguous questions, or dialogue sessions that are not convergent or too long, and so on;

- carry out monitoring interactions between the user and the chatbot, producing synthetic quality indicators and highlighting critical aspects useful for the improvement of the system.

The core of the architecture is the Inference Engine, which includes text analysis and context extraction. It is assumed that the text generated by a chat is a mixture of contextual information and that the use of some words helps to define the different context elements useful in the search for the same context that can be identified through the Context Dimension Tree (CDT) [32].

Latent Dirichlet Allocation is a model suitable for this purpose as it can be used to explain the correlation between keywords and topics (in our case, context elements), as shown in the following figure 2. Through textual analysis it is possible to know more about the student, where he is or where he would like to be (museum, archaeological park, etc.), the learning purpose of his visit and what they need.

In practice, the interaction of the user with the chatbot is divided into shorter and simpler sentences (clusters), through appropriate Bayesian filters for keywords, assuming that each sentence is semantically related to the other. The proposed approach is therefore based on the following assumption: the probability that the word W belongs to the concept node $N_{c}$ within the CDT is proportional to how much the argument (for example, the purpose of a tourist's visit to a city of art) has already been treated and the number of times that a word has been used for a specific topic. The application of this model provides a characterization of chats in an automatic way, without the needing of specify the semantic value of the words in the text.

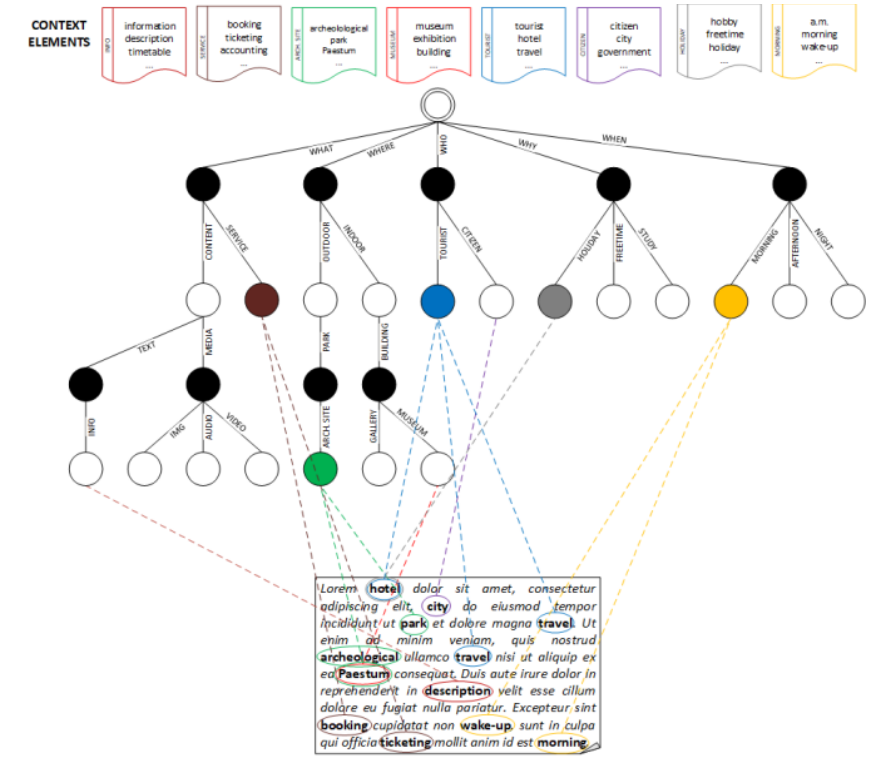

Fig. 2. Context Definition

Furthermore, using the LDA approach on a set of chats that belong to the same domain (in the case analysed, tourism), it is possible to automatically extract a Mixed Graph of Terms (mGT) that can be used both for the design of the tree of context and the constraints associated with it is to detect the context extracted in real time from the user's chat with the bot.

In particular, LDA was mainly used to generate topics within chats (text documents). These topics were processed by the system as contextual elements suitable during the use of the Context Dimension Tree. According to the LDA model, a distribution of terms for each topic $i$ is represented as a multinomial distribution $\varphi_{i}$ drawn from a symmetric Dirichlet distribution with parameter $\beta$ :

$$
p\left(\phi_{i} \mid \beta\right)=\frac{\Gamma(W \beta)}{[\Gamma \beta]^{W}} \prod_{v=1}^{W} \emptyset_{i v}^{\beta-1}
$$

The topic distribution for a document $d$ is also represented as a multinomial distribution $\Theta_{d}$ drawn by a Dirichlet distribution with parameter $\alpha$ :

$$
p\left(\theta_{d} \mid \alpha\right)=\frac{\Gamma\left(\sum_{i=1}^{K} \alpha_{i}\right)}{\prod_{i=1}^{K} \Gamma\left(\alpha_{i}\right)} \prod_{i=1}^{K} \emptyset_{d i}^{\alpha_{i}-1}
$$

In this way, the topic $z_{d n}$ for each index $n$ token can be chosen from the distribution of the document topics as:

$$
p\left(z_{d n}=i \mid \theta_{d}\right)=\theta_{d i}
$$

Each token $\mathrm{w}$ is chosen from a multinomial distribution associated with the selected topic:

$$
p\left(z_{d n}=i \mid \theta_{d}\right)=\theta_{d i}
$$

LDA aims to find patterns of co-occurrence terms in order to identify consistent topics. Through LDA it is possible to learn a topic $i$, if $p(w=v \mid z=i)$ is high for a certain term $\mathrm{v}$, then every document $d$ that contains the term $v$ has a high probability for the topic $i$.

It is possible to state that all the terms co-occurring with the term $\mathrm{v}$ are more likely to have been generated by the topic $i$. 
A complex structure like the mGT can allow to capture and represent the contextual information contained in a set of chats that belong to a specific domain (for example, tourism). This graph can be extracted automatically and used for the classification of the text, or to label the $N_{c}$ concept nodes and know which of the nodes participate in the definition of the context at a given time. Formally, it can be defined as a graph $g=\langle N, E\rangle$ where:

- $\quad N=\{R, W\}$ is a finite set of nodes, those elements can be aggregates or aggregators.

- $E=\left\{E_{R R}, E_{R W}\right\}$ is a set of arcs that connect the aggregates and aggregators.

The proposed approach is essentially composed of two basic modules located within the Inference Engine: a module for the construction of the Mixed Graph of Terms and a module for extracting the context elements.

Mixed Graph of Terms building module: this module builds the mGT starting from a set of documents that belong to a specific domain (tourism) and that have been previously labeled in accordance with the contextual information contained. The mGT can also be used in the design phase of the Context Dimension Tree.

Context Mining Module: this module involves the extraction of the context, or rather of the different context elements, thanks to the use of mGT as a context filter. The input of this module consists of a generic chat, the mGT extracted and the CDT in relation to the specific domain. The output is the context related to the chat.

Each context element is associated with a dedicated section of the database, which contains relevant and specific data. The contextual query is performed automatically by defining a global view given by the composition of the associated partial views. In addition to simple data, the same mechanism can be used for the selection of useful services related to the context identified, as shown in figure 3 .

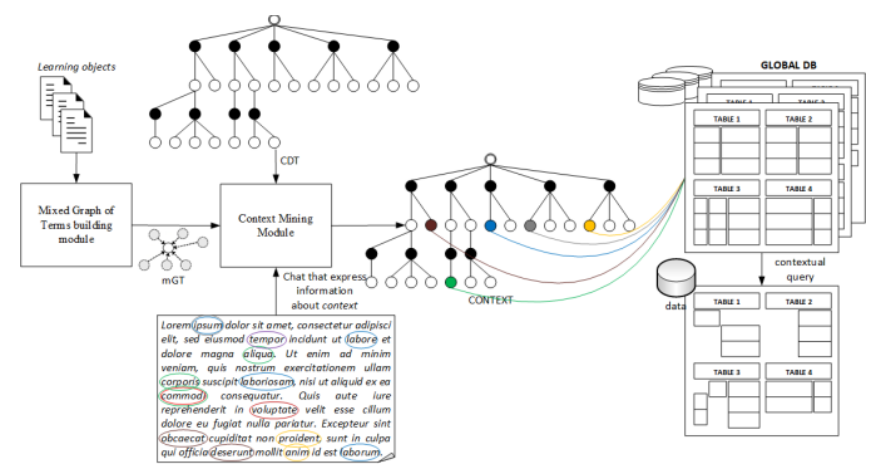

Fig. 3. Contextual queries

\section{EXPERIMENTAL RESULTS}

Based on the proposed architecture, an application prototype, implemented in Java, was developed: a chatbot, designed and implemented, along with a server-side component, as described above (figure 4). The chatbot was initially designed to support students visiting a the cultural site of the Campania region of Italy (the Archaeological parks of Paestum). In this experimental phase, the main services and contents potentially useful for students have been identified.

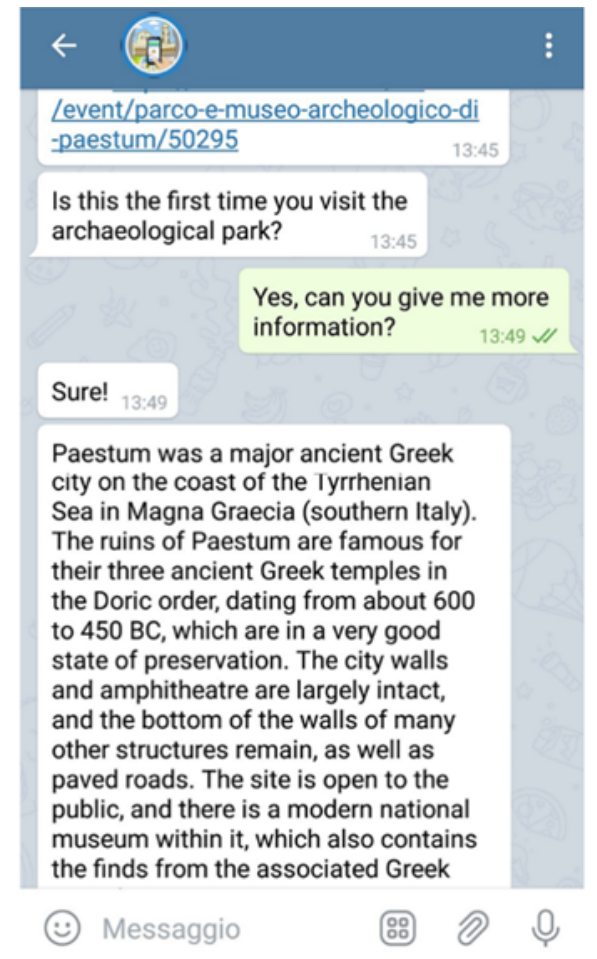

Fig. 4. The chatbot prototype at work

After the interaction with the chatbot, 73 students of the last year of the High School responded, according to the Likert scale, to a questionnaire comprising five sections. To each statement, present in a specific section, five possible answers were associated: "I totally disagree" - TD, "I disagree" - D, "Undecided" - U, "I agree" - A, "I totally agree" - TA.

Section A: recommendation

1. The proposed services and contents have satisfied the needs of the user, based on personal preferences and the current context.

2. The system has furnished effective contents for learning.

Section B: conversation

1. The dialogue with the chatbot took place smoothly and without unexpected jumps.

2. The system was able to correctly understand the intentions of the user.

Section C: presentation

1. The information has been presented appropriately.

2. The information provided was exhaustive.

Section D: usability

1. The chatbot interface is user-friendly.

2. Response times are adequate.

Section E: future developments

1. It would be useful to include in the chat other students

2. It would be interesting to apply the same approach in other scenarios.

According to Table 1, the positive responses (Agree and Totally Agree) reach a percentage always higher than $84 \%$.

TABLE I. ANALYSIS OF RESULTS

\begin{tabular}{llll}
\hline & \multicolumn{3}{c}{ Percentage } \\
\hline Section & Negative & Neutral & Positive \\
A & $7,52 \%$ & $4,87 \%$ & $87,61 \%$ \\
B & $8,01 \%$ & $6,11 \%$ & $85,88 \%$ \\
\hline
\end{tabular}




\begin{tabular}{llll}
\hline C & $8,93 \%$ & $3,72 \%$ & $87,35 \%$ \\
D & $11,12 \%$ & $3,95 \%$ & $84,93 \%$ \\
E & $7,21 \%$ & $3,05 \%$ & $89,74 \%$ \\
\hline
\end{tabular}

The lowest number of positive feedbacks is found in the conversation section: some users have found it difficult to converse fluently with the chatbot, encountering some problems related to understanding the requests made.

This can be largely due to grammatical errors or dialect words not yet learned by the system in this initial state. On the other hand, section A, relating to the context-based recommendation form, shows very satisfactory results: the services and content proposed have satisfied the needs of the user, dynamically adapting to the current context. The results therefore suggest. In particular, attention to machine learning in order to improve human-machine interaction.

\section{CONCLUSIONS}

In this paper, a chatbot for supporting students in the Cultural Heritage learning has been introduced. This chatbot adopts a natural language processing methodology based on Latent Dirichlet Allocation and Context Awareness. The proposed approach has been applied in a real scenario. The obtained results are promising. Future works aims to apply the proposed approach in other scenarios and to develop more sophisticated approach for making the conversation more "human oriented".

\section{REFERENCES}

[1] R. Winkler and M. Soellner, "Unleashing the Potential of Chatbots in Education: A State-Of-The-Art Analysis," Acad. Manag. Proc., 2018 DOI:10.5465/ambpp.2018.15903abstract.

[2] N. Albayrak, A. Ozdemir, and E. Zeydan, "An overview of artificial intelligence based chatbots and an example chatbot application," in 26th IEEE Signal Processing and Communications Applications Conference, SIU 2018, 2018 DOI:10.1109/SIU.2018.8404430.

[3] F. Clarizia, F. Colace, M. Lombardi, F. Pascale, and D. Santaniello, "Chatbot: An education support system for student," in Lecture Notes in Computer Science (including subseries Lecture Notes in Artificial Intelligence and Lecture Notes in Bioinformatics), 2018, vol. 11161 LNCS, pp. 291-302 DOI:10.1007/978-3-030-01689-0_23.

[4] A. Xu, Z. Liu, Y. Guo, V. Sinha, and R. Akkiraju, "A New Chatbot for Customer Service on Social Media," in Proceedings of the 2017 CHI Conference on Human Factors in Computing Systems - CHI '17, 2017, pp. 3506-3510 DOI:10.1145/3025453.3025496.

[5] M. Casillo, F. Clarizia, F. Colace, M. Lombardi, F. Pascale, and D. Santaniello, "An Approach for Recommending Contextualized Services in e-Tourism," Information, vol. 10, no. 5, p. 180, May 2019 DOI:10.3390/info10050180.

[6] M. Casillo, F. Clarizia, G. D’Aniello, M. De Santo, M. Lombardi, and D. Santaniello, "CHAT-Bot: a Cultural Heritage Aware Teller-Bot for supporting touristic experiences," Pattern Recognit. Lett., Jan. 2020 DOI:10.1016/j.patrec.2020.01.003.

[7] L. Benotti, M. C. Martínez, and F. Schapachnik, "A Tool for Introducing Computer Science with Automatic Formative Assessment," IEEE Trans. Learn. Technol., 2018 DOI:10.1109/TLT.2017.2682084.
Zhang, "Content-Oriented User Modeling for Personalized Response Ranking in Chatbots," IEEE/ACM Trans. Audio, Speech, Lang. Process., vol. 26, no. 1, pp. 122-133, Jan. 2018 DOI:10.1109/TASLP.2017.2763243.

[9] L. N. Paschoal, M. M. de Oliveira, and P. M. M. Chicon, “A Chatterbot Sensitive to Student's Context to Help on Software Engineering Education," in 2018 XLIV Latin American Computer Conference (CLEI), 2018, pp. 839-848 DOI:10.1109/CLEI.2018.00105.

[10] G. Molnar and Z. Szuts, "The Role of Chatbots in Formal Education," in 2018 IEEE 16th International Symposium on Intelligent Systems and Informatics (SISY), 2018, pp. 000197000202 DOI: 10.1109/SISY.2018.8524609.

[11] K. Stoeffler, Y. Rosen, M. Bolsinova, and A. A. von Davier, "Gamified assessment of collaborative skills with chatbots," in Lecture Notes in Computer Science (including subseries Lecture Notes in Artificial Intelligence and Lecture Notes in Bioinformatics), 2018 DOI:10.1007/978-3-319-93846-2_64.

[12] A. Kerly, P. Hall, and S. Bull, "Bringing chatbots into education: Towards natural language negotiation of open learner models," Knowledge-Based Syst., vol. 20, no. 2, pp. 177-185, Mar. 2007 DOI:10.1016/j.knosys.2006.11.014.

[13] A. Goel, B. Creeden, M. Kumble, S. Salunke, A. Shetty, and B. Wiltgen, "Using Watson for enhancing human-computer cocreativity," in AAAI Fall Symposium - Technical Report, 2015.

[14] G. Garcia Brustenga, M. Fuertes Alpiste, and N. Molas Castells, "Briefing Paper: Chatbots in Education," Barcelona, Sep. 2018 DOI: 10.7238/elc.chatbots.2018.

[15] F. Amato, V. Moscato, A. Picariello, and F. Piccialli, "SOS: A multimedia recommender System for Online Social networks," Futur. Gener. Comput. Syst., vol. 93, pp. 914-923, Apr. 2019 DOI:10.1016/j.future.2017.04.028.

[16] F. Amato, A. Mazzeo, V. Moscato, and A. Picariello, "Semantic Management of Multimedia Documents for E-Government Activity," in 2009 International Conference on Complex, Intelligent and Software Intensive Systems, 2009, pp. 1193-1198 DOI:10.1109/CISIS.2009.195.

[17] G. D'aniello, M. Gaeta, and M. Z. Reformat, "Collective perception in smart tourism destinations with rough sets," in 2017 3rd IEEE International Conference on Cybernetics, CYBCONF 2017 - Proceedings, 2017, pp. 1-6 DOI:10.1109/CYBConf.2017.7985765.

[18] F. Clarizia, F. Colace, M. Lombardi, F. Pascale, and D. Santaniello, "Chatbot: An Education Support System for Student," Springer, Cham, 2018, pp. 291-302 DOI:10.1007/978-3-03001689-0_23.

[19] F. Clarizia, S. Lemma, M. Lombardi, and F. Pascale, "A Mobile Context-Aware Information System to Support Tourism Events," 2017, pp. 553-566 DOI:10.1007/978-3-319-57186-7_40.

[20] M. Lombardi, F. Pascale, and D. Santaniello, “An application for Cultural Heritage using a Chatbot," in 2019 2nd International Conference on Computer Applications \& Information Security (ICCAIS), 2019, pp. 1-5 DOI:10.1109/CAIS.2019.8769525.

[21] S. A. and D. John, "Survey on Chatbot Design Techniques in Speech Conversation Systems," Int. J. Adv. Comput. Sci. Appl., vol. 6, no. 7, 2015 DOI:10.14569/IJACSA.2015.060712.

[22] M. L. Mauldin, "ChatterBots, tinyMuds, and the turing test entering the loebner prize competition," in Proceedings of the National Conference on Artificial Intelligence, 1994. 
[23] F. Amato, S. Marrone, V. Moscato, G. Piantadosi, A. Picariello, and C. Sansone, "Chatbots meet ehealth: Automatizing healthcare," in CEUR Workshop Proceedings, 2017.

[24] J. Weizenbaum, "ELIZA - A Computer Program For the Study of Natural Language Communication Between Man And Machine," Commun. ACM, 1983 DOI:10.1145/357980.357991.

[25] V. di Lecce, M. Calabrese, D. Soldo, and A. Giove, "Semantic management systems for the material support of e-learning," J. ELearning Knowl. Soc., 2010.

[26] C.-W. Liu, R. Lowe, I. Serban, M. Noseworthy, L. Charlin, and J. Pineau, "How NOT To Evaluate Your Dialogue System: An Empirical Study of Unsupervised Evaluation Metrics for Dialogue Response Generation," in Proceedings of the 2016 Conference on Empirical Methods in Natural Language Processing, 2016, pp. 2122-2132 DOI:10.18653/v1/D16-1230.

[27] P. Bottoni and M. Ceriani, "SWOWS and dynamic queries to build browsing applications: On linked data," J. Vis. Lang. Comput., 2014 DOI: 10.1016/j.jvlc.2014.10.027.

[28] P. Bellini, P. Nesi, and A. Venturi, "Linked open graph: Browsing multiple SPARQL entry points to build your own LOD views," J. Vis. Lang. Comput., vol. 25, no. 6, pp. 703-716, Dec. 2014 DOI:10.1016/j.jvlc.2014.10.003.
[29] A. Isaac, R. Clayphan, and B. Haslhofer, "Europeana: Moving to Linked Open Data," Inf. Stand. Q., vol. 24, no. 2/3, p. 34, 2012 DOI:10.3789/isqv24n2-3.2012.06.

[30] F. Colace, M. De Santo, M. Lombardi, and D. Santaniello, "CHARS: a Cultural Heritage Adaptive Recommender System," in Proceedings of the 1st ACM International Workshop on Technology Enablers and Innovative Applications for Smart Cities and Communities - TESCA'19, 2019, pp. 58-61 DOI: $10.1145 / 3364544.3364830$.

[31] F. Clarizia, F. Colace, M. De Santo, M. Lombardi, F. Pascale, and D. Santaniello, "A Context-Aware Chatbot for Tourist Destinations," in 2019 15th International Conference on SignalImage Technology \& Internet-Based Systems (SITIS), 2019, pp. 348-354 DOI:10.1109/SITIS.2019.00063.

[32] F. Colace, M. De Santo, M. Lombardi, F. Pascale, D. Santaniello, and A. Tucker, "A Multilevel Graph Approach for Predicting Bicycle Usage in London Area," in Fourth International Congress on Information and Communication Technology. Advances in Intelligent Systems and Computing, vol 1027, Y. XS., S. S., D. N., and J. A, Eds. Springer, Singapore, 2020, pp. 353-362 DOI:10.1007/978-981-32-9343-4_28. 